\title{
Modelling of microbubble coalescence
}

\author{
Y. Yonemoto ${ }^{1} \&$ T. Kunugi $^{2}$ \\ ${ }^{1}$ Priority Organization for Innovation and Excellence, \\ Kumamoto University, Japan \\ ${ }^{2}$ Department of Nuclear Engineering, Kyoto University, Japan
}

\begin{abstract}
The mechanism of bubble coalescence/repulsion is still unclear and is a key issue when it comes to numerical research on multiphase flows. Experimental studies have revealed that not only the concentration of the electrolytes dissolved in water but also the existence of ion pairs play an important role in bubble coalescence, which indicates that the contamination at the interface has an important effect on bubble interaction. In the present study, a coalescence model is developed; it is based on the conventional nucleation theory correlated with the electric conductivity originating due to the contamination of the liquid. Simple microbubble interactions are simulated using the coalescence model. Finally, we succeed in simulating the duration of contact before the coalescence of two microbubbles.
\end{abstract}

Keywords: multiphase flow, interfacial phenomena, microbubble, nucleation theory, coalescence.

\section{Introduction}

The interfacial phenomena occurring at gas-liquid interfaces are very important in various scientific fields. However, modeling gas-liquid interfaces over a wide range of scales spanning molecular motion to vortical fluid motion is very difficult, and this has remained one of the key unresolved issues in multiphase flow science and engineering since a long time. In particular, the mechanism for bubble coalescence/repulsion behaviour is unknown, although it is a superficially simple behaviour.

Numerical analysis of multiphase flow is generally performed by combining an interfacial model with the Navier-Stokes (NS) equation. The continuum surface force (CSF) model [1] is a popular interfacial model for examining gas- 
liquid interfaces. Surface tension is incorporated into the NS equation as a body force across the interface. The volume of fluid (VOF) method [2] is used with the CSF model as the interface tracking method. In the VOF method, the interface is located on the basis of the fractional volume of the fluid, given by the density fraction. Matsumoto et al. [3] performed a numerical simulation of bubbly flow on the basis of the VOF method using the CSF model. Their study showed that bubble coalescence occurs easily. It is known that the behaviour of bubble coalescence in experimental systems is not as simple as that predicted by numerical simulations. Such non-realistic bubble coalescence is estimated because of the feature of the CSF model; this model is incapable of considering the various physical and chemical processes at the gas-liquid interface because it is derived only on the basis of mechanical energy balance and assumes that the gas-liquid interface has no thickness (i.e. it is a mathematical interface).

Experimental research on bubble coalescence/repulsion mainly focuses on the effects of the concentration of the electrolyte on the behaviour and coalescence threshold of the bubbles in the liquid [4-7]. These researches reveal that the ions adsorbed at the gas-liquid interface, the combination of these ions, and electrolyte concentration are important factors in bubble coalescence/repulsion. Contamination at the interface could greatly influence bubble coalescence /repulsion because these contaminations may be related to the heat and mass transfer and electrochemistry around the interface $[8,9]$. In our previous studies $[10,11]$, a thermodynamic and mathematical interfacial model was developed on the basis of the phase field theory [12] and van der Waals theory [13], both of which assume the interface to be a diffuse interface of finite thickness. On the basis of our new interfacial model, the multi-scale multiphase flow equation was applied to simple numerical simulation of micro-bubble interaction [14].

In the present study, the coalescence model is developed by associating the conventional nucleation theory [15] with the electrical conductivity originating from the contamination of the liquid. Microbubble interaction is evaluated using the coalescence model, and mass transfer is considered in the numerical simulation. As a result, the duration of contact before the coalescence of two microbubbles is simulated using the coalescence model.

\section{Multi-scale modeling of gas-liquid interface}

In our previous study [10], an interfacial model was developed and a multi-scale multiphase flow equation was derived on the basis of the phase field theory. The modeling process is now briefly reviewed.

For the purpose of modeling, we assumed that the interface has a finite thickness as does a fluid membrane, as shown in figure 1 (development of mesoscopic interface). It was found that the contamination at the gas-liquid interface may be an important factor in experimental observations of bubble interaction [5, 6]. Accordingly, the contamination at the interface is associated with an electrostatic potential owing to the presence of an electric double layer at the interface. The free energy equation including the electrostatic potential is derived on the basis of a lattice gas model [16], 


$$
F=\int_{V}\left[f_{0}(\psi)+\frac{d}{2}(\nabla \psi)^{2}\right] \mathrm{d} V
$$

Here

$$
\begin{gathered}
f_{0}(\psi)=-\frac{a}{2} \psi^{2}+\frac{b}{4} \psi^{4}-c Z_{i} e V_{e} \psi \\
a=\frac{U_{\text {sum }}-4 k_{B} T}{l^{3}} \\
b=\frac{16 k_{B} T}{3 l^{3}} \\
c=\frac{1}{2 l^{3}} \\
d=\frac{U_{\text {sum }}}{2 l^{3}}
\end{gathered}
$$

In eqn. (1), the first and second terms represent the free energy in homogenous and inhomogeneous systems, respectively. Here, the coefficients $a\left[\mathrm{~J} / \mathrm{m}^{3}\right], b\left[\mathrm{~J} / \mathrm{m}^{3}\right], c\left[1 / \mathrm{m}^{3}\right]$, and $d[\mathrm{~J} / \mathrm{m}]$ in eqns. (1) and (2) have constant values. In eqns. (3) and (6), $U_{\text {sum }}$ is related to an intermolecular potential. These values include the microscopic information. The symbols $z_{\mathrm{i}}[-], e[\mathrm{C}], V_{\mathrm{e}}[\mathrm{V}]$, and $\psi[-]$ denote the charge number, elementary charge, electrostatic potential, and order parameter, respectively. The order parameter characterizes a system. The third term on the right-hand side of eqn. (2) is related to the contamination at the interface.

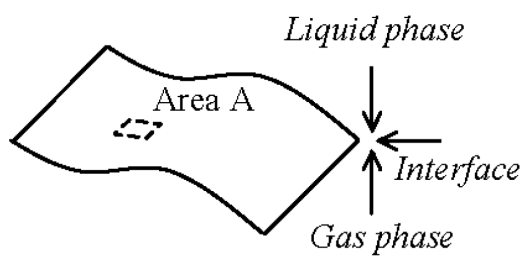

(a)

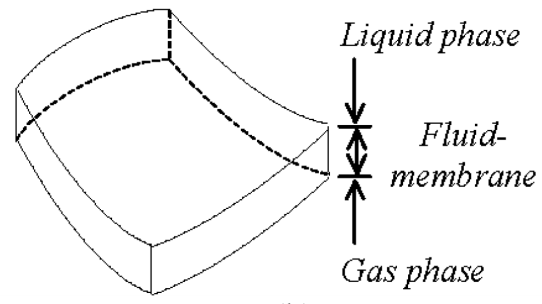

(b)

Figure 1: Concept of gas-liquid interface: (a) macroscopic image and (b) mesoscopic image of Area A.

Many physical and chemical processes, which are characterized by various time and space scales, occur at the interface. Therefore, in order to consider the interactions occurring on various scales, from the microscopic to the macroscopic scale, the Chapman-Enskog expansion [17] is applied to the NS equation. Here, the original NS equation is expressed as follows:

$$
\rho \frac{\mathrm{D} \boldsymbol{u}}{\mathrm{D} t}=-\nabla \cdot \boldsymbol{T}+\rho \boldsymbol{g}
$$


in which the symbols $\rho\left[\mathrm{kg} / \mathrm{m}^{3}\right], \boldsymbol{u}[\mathrm{m} / \mathrm{s}], \boldsymbol{T}\left[\mathrm{N} / \mathrm{m}^{2}\right], \boldsymbol{g}\left[\mathrm{m} / \mathrm{s}^{2}\right]$, and $t[\mathrm{~s}]$ represent the fluid density, velocity, stress tensor, acceleration of gravity, and time, respectively. At this point, the operators $\mathrm{D} / \mathrm{D} t$ and $\nabla$ must include multiscale components. Therefore, in order to discriminate between their scales, the Chapman-Enskog expansion is applied to $\mathrm{D} / \mathrm{D} t$ and $\nabla$ in the NS equation. The expansion of the operators $\mathrm{D} / \mathrm{D} t$ and $\nabla$ by a small parameter $\varepsilon$ gives

$$
\begin{gathered}
\nabla=\nabla^{(0)}+\varepsilon \nabla^{(1)}+\varepsilon^{2} \nabla^{(2)}+\cdots+\varepsilon^{k} \nabla^{(k)}+\cdots \\
\frac{\mathrm{D}}{\mathrm{D} t}=\frac{\mathrm{D}}{\mathrm{D} t^{(0)}}+\varepsilon \frac{\mathrm{D}}{\mathrm{D} t^{(1)}}+\varepsilon^{2} \frac{\mathrm{D}}{\mathrm{D} t^{(2)}}+\cdots+\varepsilon^{k} \frac{\mathrm{D}}{\mathrm{D} t^{(k)}}+\cdots
\end{gathered}
$$

where the superscript $(k)(k=0,1,2, \ldots)$ represents the scale of the phenomena. The scale becomes small with an increase in the value of $(k)$. For example, the superscript (0) indicates the macroscopic scale. In eqns. (8) and (9), the small parameter $\varepsilon$ is defined as $\varepsilon=\delta / L$ where the symbols $\delta$ [m] and $L[\mathrm{~m}]$ represent the characteristic length of the interface and that of the vortical fluid flow, respectively. After the substitution of eqns. (8) and (9) into eqn. (7) and simple tensor analysis, a new governing equation is obtained as follows:

$$
\varepsilon \rho \frac{\mathrm{D} \boldsymbol{u}^{\prime}}{\mathrm{D} t^{(1)}}=-\nabla^{(0)} \cdot \boldsymbol{T}+\varepsilon \boldsymbol{\nabla}^{(1)} \cdot\left(f_{0}(\psi) \boldsymbol{I}\right)-\varepsilon d \nabla^{(1)} \psi\left(\nabla^{(1)} \cdot \nabla^{(1)} \psi\right)+\rho \boldsymbol{g} .
$$

In the derivation, the free energy (eqn. (1)) is associated with thermodynamic pressure by using the Maxwell relation [10]. Equation (10) is the multi-scale multiphase flow equation. Our interfacial model has a theoretical multi-scale concept from a microscopic to a macroscopic scale because the interfacial jump condition that characterizes the macroscopic interface is derived on the basis of eqn. $(10)[11,18]$.

\section{Numerical simulation}

\subsection{Governing equation}

In the present study, chemical reactions and phase changes are not considered, which means that the time and space interactions in each phenomenon are not considered. Thus, the microbubble behavior in the local regions around the microbubble is considered and simulated: all of the space scales in eqn. (10) are treated as the mesoscopic scale $\left(\nabla^{(1)}\right)$. Subsequently, we do not distinguish the velocity vectors and combine the two terms appearing on the left-hand side of eqn. (10) into D/Dt . Finally, the following simplified equations are applied to the present qualitative numerical simulation.

$$
\begin{gathered}
\rho \frac{\mathrm{D} \boldsymbol{U}}{\mathrm{D} t}=-\boldsymbol{\nabla}^{(1)} \cdot \boldsymbol{T}+\varepsilon \boldsymbol{\nabla}^{(1)} \cdot\left(f_{e}(\psi) \boldsymbol{I}\right)-\varepsilon d \boldsymbol{\nabla}^{(1)} \psi\left(\boldsymbol{\nabla}^{(1)} \cdot \boldsymbol{\nabla}^{(1)} \psi\right)+\rho \boldsymbol{g}, \\
\boldsymbol{\nabla}^{(1)} \cdot \boldsymbol{U}=0
\end{gathered}
$$


With respect to the second term on the right-hand side of eqn. (1), only the electric term $f_{e}=c z_{i} e V_{e} \psi$ is considered: the first and second terms in eqn. (2) are omitted in eqn. (1) because the phase change is not considered.

In our interfacial model, the existence of the contamination at the interface is represented by the existence of a molecule $\mathrm{A}$ or $\mathrm{B}$. That is, the electrostatic potential due to the contamination at the interface in the bulk phase A (or B) is valid when $F_{\mathrm{A}}=1$ (or $F_{\mathrm{B}}=1$ ). The concrete formula for $f_{\mathrm{e}}$ is expressed as $f_{e}=\left.c z_{i} e V_{e}\right|_{i} \psi_{e}$. In the strict sense, the Maxwell equations must be applied to $f_{\mathrm{e}}$ in order to understand the relation between the electric charge and electric potential. However, the law of conservation of charge is not considered in this study for the sake of simplicity. Therefore, in the bulk phases A and B, the electrostatic potential $\left.V_{e}\right|_{i}$ at each interface is determined independently. The electrostatic potential of $\left.V_{e}\right|_{i}$ is estimated by the following exponent function:

$$
V_{e}(\boldsymbol{x})=V_{e 0} \exp \left(-\frac{\left|\boldsymbol{x}-\boldsymbol{x}_{\text {surface }}\right|}{C_{e}}\right)
$$

In this equation, the symbol $C_{\mathrm{e}}$ is an arbitrary parameter that determines the effective region of the electrostatic potential, such as the Debye length. The position $\boldsymbol{x}$ is arbitrary, except for the region inside a bubble. The position $\boldsymbol{x}_{\text {surface }}$ is the surface position of the bubble. The surface position is determined by the labeling method [3]. The substitution of $f_{e}=\left.c z_{i} e V_{e}\right|_{i} \psi_{e}$ into the second term on the right-hand side of eqn. (11) gives the electrostatic force $F_{\mathrm{e}}$ as follows:

$$
\boldsymbol{F}_{e}=-\varepsilon c z_{i} e \psi_{e} \nabla^{(1)}\left(\left.V_{e}\right|_{i}\right)
$$

where $z_{\mathrm{i}} e$ denotes the charge of the contamination adsorbed at the interface. This value is defined at the surface position. When an interfacial interaction is considered between bubbles 1 and 2, the electrostatic force is calculated by using the charge $z_{\mathrm{i}} e$ and the electrostatic potential $V_{\mathrm{e}}$. For example, the electrostatic force at the interface of bubble 1 is estimated by the charge $z_{\mathrm{i}} e$ defined at the surface position of bubble 1 and the electrostatic potential $V_{\mathrm{e}}$ from bubble 2 .

\section{Coalescence model}

Many pieces of experimental evidence reveal the importance of the effect of electrolytes and adsorbed ions at the interface with respect to bubble coalescence [4-7]. This indicates that mass transfer may be related to interfacial interactions. According to Henry's law, the amount of a gas dissolved in a liquid is proportional to the pressure of the gas in equilibrium when the liquid is at a constant temperature. Similar laws may be applied to the region around the bubble interface in a liquid. In the present numerical simulation, one of the possibilities of bubble coalescence is modeled by focusing on a relationship, 
based on Henry's law, between the contamination in the liquid and the dissolved gas in a region around the bubble interface.

\subsection{Nucleation theory}

Bubble coalescence is modeled on the basis of a conventional nucleation theory [15]. Consider a situation that two bubbles approach each other and touch each other. A liquid film forms between the two bubbles. Here, we assume that tiny bubbles arise in the liquid film via the contamination in the bulk liquid; the contamination acts as the bubble nucleus. The source of the tiny bubbles is the dissolved gas near the bubble interfaces in the liquid film. The generated tiny bubbles may cause bubble coalescence. Moreover, in the nucleation process, we assume that the contamination related to the generation of tiny bubbles is different from that responsible for the electrostatic potential at the bubble interface and has no relation with the electrostatic potential.

In the conventional homogeneous nucleation theory, nucleation generally occurs in a uniform and supersaturated gas or solid phase. The gas or solid molecules aggregate and form a spherical cluster. Then, if the radius of the cluster exceeds the critical radius calculated from the Gibbs free energy and the change in the chemical potential of the spherical cluster, the cluster grows until the thermodynamic equilibrium is attained. The following equations are for the Gibbs free energy, chemical potential, and critical radius.

$$
\begin{gathered}
\Delta G=-\frac{4}{3} \pi r^{3} \frac{1}{v_{0}} \Delta \mu+4 \pi r^{2} \sigma \\
\Delta \mu=k_{B} T \ln \frac{P_{g A}}{P_{g B}} \\
r_{c r i t}=\frac{2 \sigma v_{0}}{\Delta \mu}
\end{gathered}
$$

Equation (17) gives the criterion that determines whether the nucleated cluster grows up or not. This theory is mainly used in studies on nucleation from a gas to a solid or liquid. However, we apply this theory to dissolved gas molecules in a liquid. That is, we assume that the dissolved gas molecules aggregate and form a tiny bubble. In the nucleation process, gas molecules are treated as solute molecules that do not interact with the liquid (solvent) molecules. In general, a liquid contains some contaminations, even if it is purified. It is very difficult to qualitatively identify individual contaminations. However, their amount can be estimated by measuring the electric conductivity of the liquid. Thus, a new heterogeneous nucleation theory is developed based on the homogeneous nucleation theory by considering the electric conductivity of the liquid.

Figure 2 shows two bubbles A and B interacting in a liquid. First, as shown in fig. 2(a), consider situation 1 before the nucleation, where there are bubbles $A$ and B and some contaminations. The dissolved gas, according to Henry's law, exists in the liquid near the bubble interfaces, and the gas-saturated area is around the bubbles. Figure 2(b) shows situation 2 after the nucleation, where a 


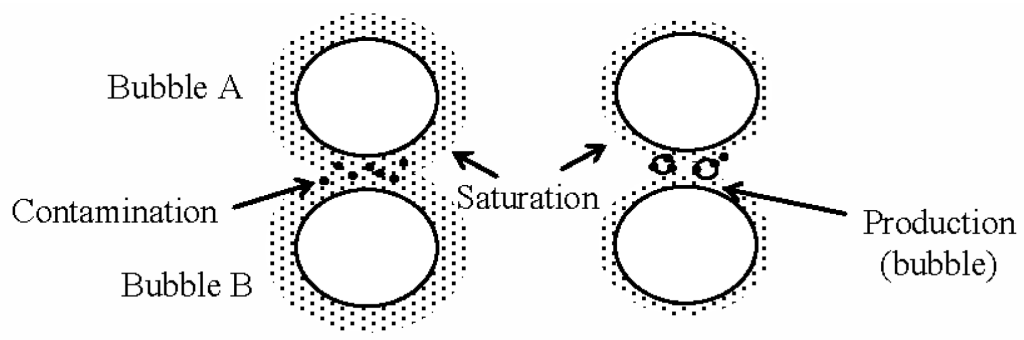

(a) Before

(b) After

Figure 2: Bubble production between two bubbles: (a) Before (Situation 1), (b) After (Situation 2).

tiny bubble generates in the liquid film via the contaminations. The saturation solubility decreases depending on the flow field in the liquid film and around the bubbles, which results in the formation of a supersaturated region of the dissolved gas in the liquid film. The critical radius in this situation is calculated by considering the change in the chemical potential from situation 1 to 2 . The change in the chemical potential is obtained as follows:

$$
\Delta \mu=k_{B} T\left(\ln \frac{P_{g A}}{P_{g B}}+\ln \frac{C_{\text {dust }}}{C_{\text {pure }}}\right)
$$

where the subscripts "dust" and "pure" imply the contaminated and pure liquid (i.e., with few contaminations), respectively. The symbols $C_{\text {dust }}$ and $C_{\text {pure }}$ denote the concentrations of the contaminations. Here, we assume that the electric conductivity of the liquid is proportional to the concentrations if the concentrations are small: $\Omega_{i}=k C_{i}$ In this numerical simulation, we set $\Omega_{\text {pure }}=1$ $[\mu \mathrm{S} / \mathrm{cm}]$, which is the value for pure water. Moreover, an arbitrary parameter $\beta_{0}$, which compensates the difference between the theory and experiment is introduced in eqn. (18). Finally, equation (18) is transformed into the following equation:

$$
\Delta \mu=k_{\mathrm{B}} T\left(\ln \frac{P_{g A}}{P_{g B}}+\beta_{0} \ln \Omega_{\text {dust }}\right)
$$

The nucleation of tiny bubbles is judged on the basis of the critical radius derived by substituting eqn. (19) in eqn. (17). In addition, we assume that the nucleation occurs in a region where the pressure is lowest in the liquid film. The generation of tiny bubbles is judged by comparing the critical radius $r_{\text {crit }}$ with an averaged radius $r_{\text {ave }}$, which is calculated from the amount of diffused gas in each cell. 


\section{Numerical condition}

In the present study, three dimensional (3D) numerical simulations are performed for the interaction between two microbubbles. In the simulation, equations (11) and (12) are solved by the projection method [11]. The interface is tracked by the multi-interface advection and reconstruction solver (MARS) method [19]. This method is similar to the piecewise linear interface construction (PLIC) [20] algorithm and is based on the volume of fluid (VOF) method [2]. The physical properties considered are those of water and air. Figure 3 shows the $3 \mathrm{D}$ numerical domain. The size of the domain is $115 \times 101 \times 61$. The boundary conditions are that the pressure free at $\mathrm{X}-$ and $\mathrm{X}+$ and slip occurs at $\mathrm{Z}$ - and $\mathrm{Z}+$. At $\mathrm{Y}-$ and $\mathrm{Y}+$, the velocities are kept constant, i.e., $\left(U_{x}, U_{y}, U_{z}\right)=(0$, $\left.1.0 \times 10^{-3}, 0\right)$ at $\mathrm{Y}+$ and $\left(0,+1.0 \times 10^{-3}, 0\right)$ at $\mathrm{Y}-$. The bubble radii, mesh sizes, and the time interval are $r=1.5 \times 10^{-6}[\mathrm{~m}], \mathrm{d} x=\mathrm{d} y=\mathrm{d} z=1.0 \times 10^{-7}[\mathrm{~m}]$ and $\mathrm{d} t=$ $2.5 \times 10^{-6}$ [s]. In this simulation, we consider three cases. Two bubbles mutually interact. In case 1 , the electrostatic potential and coalescence model are not considered. In case 2, only the electrostatic potential, $V_{\mathrm{e}}=-1.0 \times 10^{-5}[\mathrm{~V}]$, is considered. In case 3, both the electrostatic potential, $V_{\mathrm{e}}=-1.0 \times 10^{-5}[\mathrm{~V}]$, and the coalescence model are considered. The parameters used in the coalescence model are $\beta_{0}=33.01[-]$ and $\Omega_{\text {dust }}=103.146[\mu \mathrm{S} / \mathrm{cm}]$.

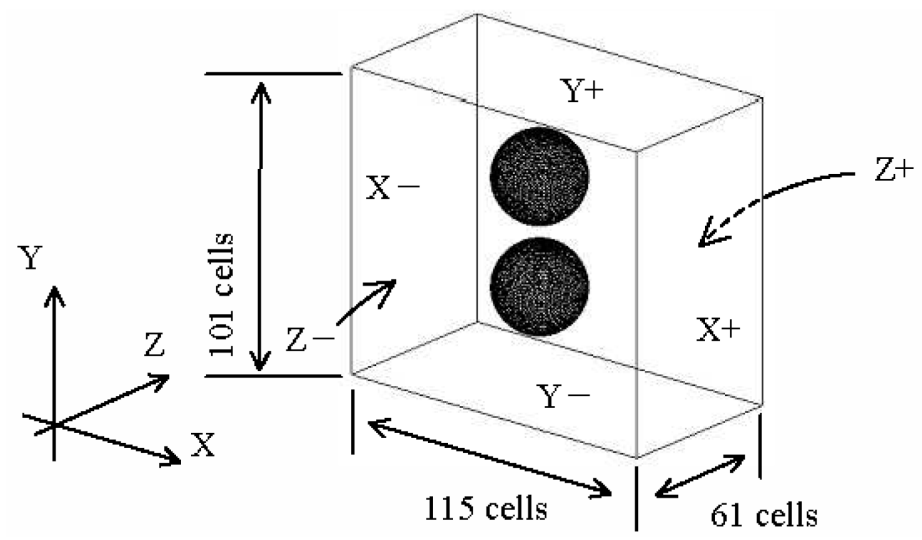

Figure 3: Computational domain of bubble interaction.

The microscopic parameters are $a=4.57 \times 10^{-8}\left[\mathrm{~J} / \mathrm{m}^{3}\right], b=5.74 \times 10^{8}\left[\mathrm{~J} / \mathrm{m}^{3}\right]$, $c=1.33 \times 10^{28}\left[1 / \mathrm{m}^{3}\right]$, and $d=4.98 \times 10^{-11}[\mathrm{~J} / \mathrm{m}]$. The multi-scale parameter is set to $\varepsilon=0.055[-]$ [14]. This parameter consists of the characteristic length $L$ and $\delta$. The characteristic length $L$ is set to $1[\mathrm{~m}]$ which corresponds to the Kolmogorov scale. $\delta$ is set to the simple average of 10 [nm] and 100 [nm], i.e., it is set to 55 [nm]. This range corresponds to the van der Waals region and is the limit thickness of the film rupture [21, 22]. 


\section{Results and discussion}

Figure 4 shows the time series of the 3D bubble interaction. Figures 4(a), (b), and (c) correspond to cases 1, 2, and 3, respectively. In fig. 4(a), neither the electrostatic potential of the bubble interface nor the coalescence model is considered. Thus, the two bubbles approach each other and simply coalesce after touching. On the other hand, figure 4(b) shows the interaction between two bubbles, in which only the electrostatic potential is considered. The two bubbles approach each other due to the hydrodynamic force from $\mathrm{Y}+$ and $\mathrm{Y}$ - directions and mutually interact. However, they flow separately while maintaining a constant distance and do not coalesce. In fig. 4(c), both the electrostatic potential and the coalescence model are considered. For the first 300 steps, the behavior of the two bubbles shown in this figure is the same as those of the ones shown in fig. 4(b). However, the two bubbles shown in fig. 4(c) coalesce after 300 steps. Obviously, the timing of coalescence in case 3 is different from that in case 1 . In case 3 , the two bubbles coalesce after a delay.

Figure 5 shows a snapshot of microbubble interaction at 400 steps in case 2 . From this figure, a clear shape of the liquid film, resembling a disk, can be observed. In the same time-step, a pressure distribution in the liquid film is lower than the bulk pressure around the microbubbles. The microbubble coalescence in fig. 4(c) may result from the generation of tiny bubbles in a region of the low pressure. Moreover, this result indicates that the bulk liquid flows into the liquid film to conserve the continuity and maintain the liquid film. The pressure distribution in the liquid film was also observed in the 2D microbubble interaction conducted by us [14]. The liquid film results from two forces. One is

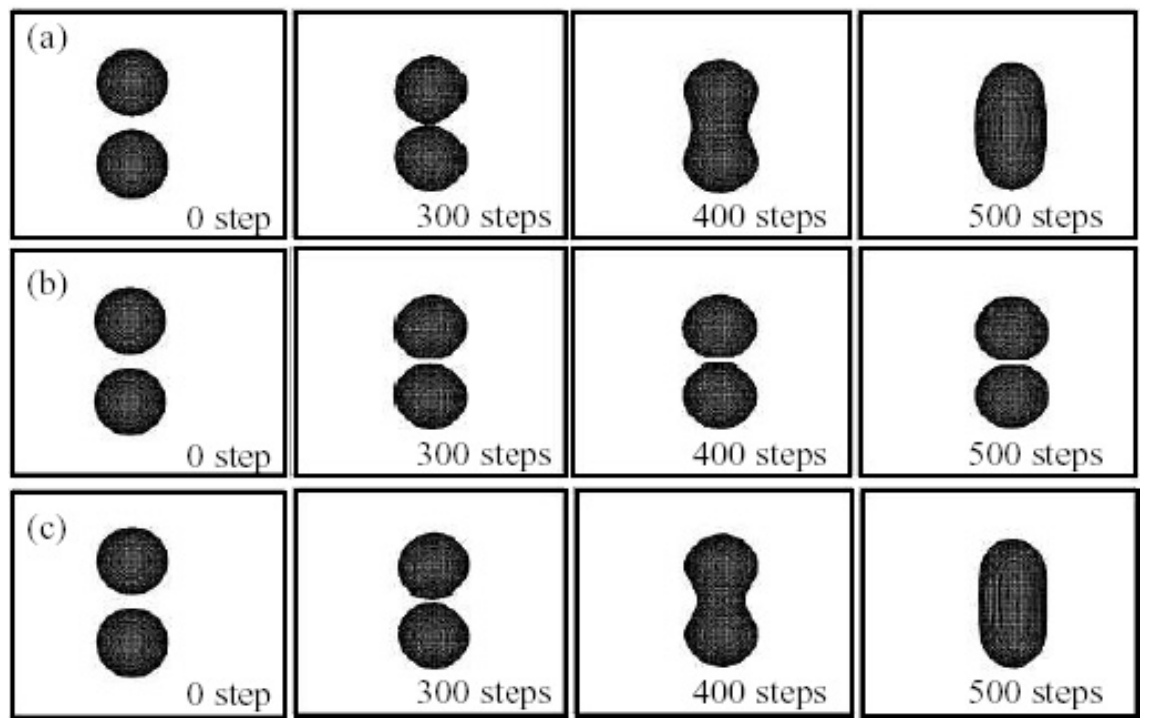

Figure 4: $\quad$ Time series of bubble interaction: (a) case 1, (b) case 2, (c) case 3. 
(a)

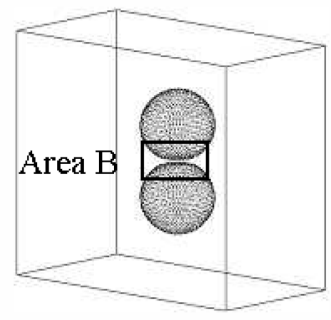

(b)

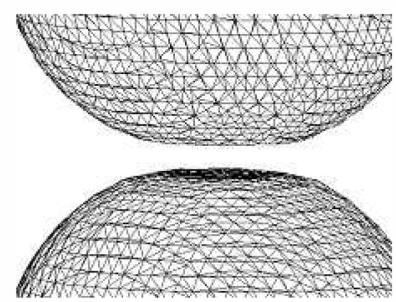

Figure 5: $\quad$ Three dimensional liquid film at 400 steps in case 2: (a) birds-eye view, (b) local region (Area B).

hydrodynamic force which cases two microbubbles to approach each other. The other is electrostatic force which cases two micobubbles to be separated. Thus, it can be seen that the present 3D numerical results support our previous conclusion [14] that the lubrication theory [23], which is based only on hydrodynamics, cannot explain the premise of the existence of a liquid film between two microbubbles. In particular, the liquid film cannot be observed in case 1 , where the electrostatic potential is not considered.

\section{Conclusions}

In this study, three-dimensional numerical simulation of microbubble interaction is performed on the basis of a multi-scale multiphase flow equation. In the simulation, a model of microbubble coalescence is considered in addition to the electrostatic potential. Finally, the following results are obtained:

1. The model of microbubble coalescence is developed on the basis of the conventional nucleation theory. The newly developed coalescence model is associated with electric conductivity, which expresses the measure of the contamination in the liquid. The electric conductivity is related to the concentration of the contamination and determines the critical radius of nucleation.

2. Numerical simulation of 3D microbubble interactions with the coalescence model is performed. The microbubbles simply coalesce with each other in case 1 where neither the coalescence model nor the electrostatic potential is considered. Then, in case 2, where only the electrostatic potential is considered, a liquid film can be observed between two microbubbles, and the microbubbles are observed to flow while maintaining a constant distance. In this case, the microbubbles do not coalesce. In case 3, where both the coalescence model and the electrostatic potential are considered, two microbubbles coalesce with each other after some duration of contact. Therefore, the present coalescence model in which the conventional nucleation theory is combined with the electrostatic potential at the interface can explain the differences in the timing of microbubble coalescence.

The present numerical results indicate the possibility that bubble interactions on the mesoscopic scale can be simulated by using the multi-scale multiphase flow equation. Further, our interfacial model does not perfectly consider the 
various physical phenomena occurring at the interface. Thus, there may be some processes with respect to bubble coalescence in addition to those considered in the present coalescence model. However, at least, it can be seen that there are two coalescence processes: One process is only related to the electrostatic potential, and the other is related to mass transfer in addition to the electrostatic potential.

\section{References}

[1] Brackbill, J. U., Kothe, D. B. and Zdmach, C., Continuum method for modeling surface tension. J. Comput. Phys., 100, 335-354, 1992.

[2] Hirt, C. W. and Nichols, B. D., Volume of fluid method for the dynamics of free boundaries. J. Comput. Phys., 39, 201-225, 1981.

[3] Matsumoto, Y., Fukami, T., Kunugi, T. and Serizawa, A., Fluid-dynamic structure of air-water bubbly flow with micro-bubbles, Proc. of the 4th International Conference on Multiphase Flow, CDROM-332, 2001.

[4] Spyridopoulos, M. T. and Simons, S. J. R., Effect of natural organic matter on the stability of a liquid film between two colliding bubbles. Colloids Surf. A., 235, 25, 2004.

[5] Craig V. S. J., Bubble coalescence and specific-ion effects, Curr. Opin. Colloid Interface Sci. 9, 178, 2004.

[6] Henry, C. L. and Craig, V. S. J., Ion-specific influence of electrolytes on bubble coalescence in nonaqueous solvents., Langmuir 24, 7979, 2008.

[7] Marčelja, S., Short-range forces in surface and bubble interaction, Curr. Opin. Colloid Interface Sci., 9, 165, 2004.

[8] Martin, M., Montes, F. J. and Galan, M. A., Bubble coalescence at sieve plates: II. Effect of coalescence on mass transfer. Superficial area versus bubble oscillations, Chem. Eng. Sci., 62, 1741, 2007.

[9] Auster, N. D., Gunde, R., Mader, R. and Windhab, E. J., Binary coalescence of gas bubbles in the presence of a non-ionic surfactant, J. Colloid Interface Sci. 333, 579, 2009.

[10] Yonemoto, Y. and Kunugi, T., Multi-scale modeling of the gas-liquid interface based on mathematical and thermodynamic approaches, Open Transport Phenom. J., 2, 69, 2010.

[11] Yonemoto, Y. and Kunugi, T., Study on multi-scale modeling of gas-liquid interface and microbubble coalescence, The 6th International Symposium on Multiphase Flow, Heat Mass Transfer and Energy Conversion., AIP Conf. Proc. 1207, 735, 2010.

[12] Cahn, J. W. and Hilliard, J. E., Free energy of a nonuniform system. I. interfacial energy. J. Chem. Phys. 28(2), 258-267, 1958.

[13] Rowlinson, J. S. and Widom, B., Molecular theory of capillarity, Oxford University Press, 1984.

[14] Yonemoto, Y. and Kunugi, T., Fundamental numerical simulation of microbubble interaction based on multi-scale multiphase flow equation, Microgravity Sci. Technol., 22(3), pp. 397-405, 2010. 
[15] Abraham, F. F., Homogeneous nucleation theory, Academic Press, NY, 1974.

[16] Safran, S. A., Statistical thermodynamics of surfaces, interfaces, and membranes, Addison Wesley-Publishing Company, Massachusetts, 1994.

[17] Chapman, S. and Cowling, T., The mathematical theory of non-uniform gases, 3rd Edn. Cambridge University Press, Cambridge, 1970.

[18] Yonemoto, Y., Kunugi, T., Macroscopic gas-liquid interfacial equation based on thermodynamic and mathematical approaches (Chapter 4). Mass Transfer - Advanced Aspects, ed. Dr. Hironori Nakajima, InTech, pp. 59 80, 2011.

[19] Kunugi, T., MARS for multiphase calculation., Comput. fluid dynamics, 9, 563, 2001.

[20] Youngs, D. L., Numerical methods for fluid dynamics., Academic Press.,1982.

[21] Goodall, D. G., Gee, M. L., Stevens, G., Perera, J. and Beaglehole, D., An investigation of the critical thickness of film rupture and drainage phenomena using dual wavelength ellipsometry. Colloids and Surfaces, 143, 41, 1998.

[22] Witelski, T. P. and Bernoff, A. J., Dynamics of three-dimensional thin film rupture, Physica D 147, 155, 2000.

[23] Reynolds, O., On the theory of lubrication and its application to Mr. Beauchamp Tower's experiments, including an experimental determination of the viscosity of olive oil, Philos. Trans. R. Soc. Lond., 177, 157, 1886. 